\title{
Percutaneous transluminal angioplasty
} of complex aortoiliac occlusive disease secondary to Takayasu's aortoarteritis in a young female

\section{Pankaj Jariwala, Rajendra V Irlapati ${ }^{1}$, Suresh Giragani², Sikandar Shaikh³}

Departments of Cardiology, ${ }^{1}$ Rheumatology, ${ }^{2}$ Interventional Radiology and ${ }^{3}$ Radiodiagnosis, Yashoda Hospitals, Somajiguda, Raj Bhavan Road, Hyderabad, Telangana, India

Correspondence: Dr. Pankaj Jariwala, Department of Cardiology, Yashoda Hospitals, Somajiguda, Raj Bhavan Road, Hyderabad, - 500082 , Telangana, India. E-mail: Pankaj_jariwala@hotmail.com

\begin{abstract}
As the initial treatment of patients with the iliac occlusive disease, percutaneous transluminal angioplasty (PTA) became common. Though not supported by the latest TransAtlantic Inter-Society Consensus (TASC) II guidelines, percutaneous treatment of complex aortoiliac lesions is possible and provides comparable angiographic and clinical outcomes compared to open surgery at both short- and long-term follow-up, also in complex lesion settings. TASC C and D lesions with the latest instruments, procedures, and modalities may also be managed endovascularly. It provides new opportunities for a population of highly comorbid patients. We assume that the outcomes of endovascular therapy for aortoiliac lesions in the setting of Takayasu's arteritis will be further enhanced through continuous technological progress and new advances in materials. In light of the current progression towards minimally invasive procedures, a growing number of skilled centres should be able to treat by endovascular intervention the great majority of all arterial pathologies.
\end{abstract}

Key words: Aortoiliac occlusive disease; percutaneous trans-luminal angioplasty; self-expanding stents; Takayasu Arteritis

\section{Introduction}

Takayasu arteritis (TA) is an uncommon type of chronic vasculitis that has a characteristic effect on the aorta and its primary branches and is more common in young women. While not all patients have distinct stages, TA is distinguished by three stages: aggressive inflammation with constitutional symptoms, vascular inflammation with symptoms of vessel pain or tenderness, and ischemic or aneurysmal fibrosis or degeneration of the vessel. ${ }^{[1]}$

\begin{tabular}{|l|l|}
\hline \multicolumn{2}{|c|}{ Access this article online } \\
\cline { 1 - 2 } Quick Response Code: & Website: \\
\cline { 1 - 2 } & www.ijri.org \\
& DOI: \\
\cline { 1 - 2 } & 10.4103/ijri.IJRI_225_20 \\
\hline
\end{tabular}

Percutaneous intervention in the setting of the Takayasu's aortoarteritis (TA) is difficult as lesions are fibrotic and require special hardware to cross them. Repeat revascularization necessitates repeat procedures of the same or different vascular territories. Restenosis rates are very high, and intervention should be attempted in an inactive stage unless life-threatening.

This is an open access journal, and articles are distributed under the terms of the Creative Commons Attribution-NonCommercial-ShareAlike 4.0 License, which allows others to remix, tweak, and build upon the work non-commercially, as long as appropriate credit is given and the new creations are licensed under the identical terms.

For reprints contact: WKHLRPMedknow_reprints@wolterskluwer.com

Cite this article as: Jariwala P, Irlapati RV, Giragani S, Shaikh S. Percutaneous transluminal angioplasty of complex aortoiliac occlusive disease secondary to Takayasu's aortoarteritis in a young female. Indian J Radiol Imaging 2020;30:405-8.

Received: $10-A p r-2020$ Accepted: 18 -Jun-2020

Revised: 28-May-2020 Published: $15-$ Oct-2020 


\section{Case Report}

A 21-year-old female presented with complaints of progressive NYHA Class III breathlessness with episodes of paroxysmal dyspnoea and intermittent claudication Rutherford Class II for 2 months. The referring physician assumed the presence of the cardiovascular disease, as all of her four limbs had absent peripheral pulses. On examination, she was thin built and undernourished with muscle wasting of the four limbs. We could not record accurate blood pressure. Heaving apex was discovered by cardiac examination, $2 \mathrm{~cm}$ outside the midclavicular line, and laterally displaced. Grade II mid-diastolic murmur with regular heart rhythms could be heard on auscultation.

Laboratory parameters were normal except for elevated acute phase reactants. Electrocardiography demonstrated features of left ventricular hypertrophy with strain pattern like poor ' $R$ ' wave progression. Computed tomography (CT) angiography of aorta revealed the complete occlusion of the infrarenal abdominal aorta and bilateral iliac arteries. It also demonstrated the occlusion of bilateral subclavian arteries with good collateralization [Figure 1A-C]. Complete occlusion of the right renal artery and $80 \%$ stenosis of the left renal artery were also delineated. Echocardiography showed dilated left ventricle, global hypokinesia, concentric left ventricular hypertrophy, and moderate mitral regurgitation, with small apical clot [Figure 1D].

These features were suggestive of hypertensive heart disease. Based on the results of clinical examination and investigations, a diagnosis of TA was made.

Medical therapy in the form of newer oral anticoagulant, aspirin, diuretic, spironolactone, and empirical antihypertensives was started. Rheumatologist prescribed her steroids, once a weekly dose of methotrexate and mycophenolate mofetil. Her symptoms improved to NYHA class II over 2 weeks and could perform her routine activities.

After 9 months of immunosuppressive treatment, the intervention was planned for the revascularization of the lower limb vessels to alleviate their symptoms after acute phase reactants had plummeted to normal levels. The vascular surgeon recommended against surgical revascularization because the extensive dissection and high risk of graft restenosis would have been involved. The patients and their families chose less invasive solutions against the possibility of repeat revascularization.

The percutaneous intervention was planned along with interventional radiologists. Under ultrasound guidance, both groins were punctured using a micro-needle to reduce the risk of injury to the arterial wall. Digital subtraction angiography (DSA) has demonstrated the occlusion of bilateral iliac arteries. The left iliac artery had a distal end

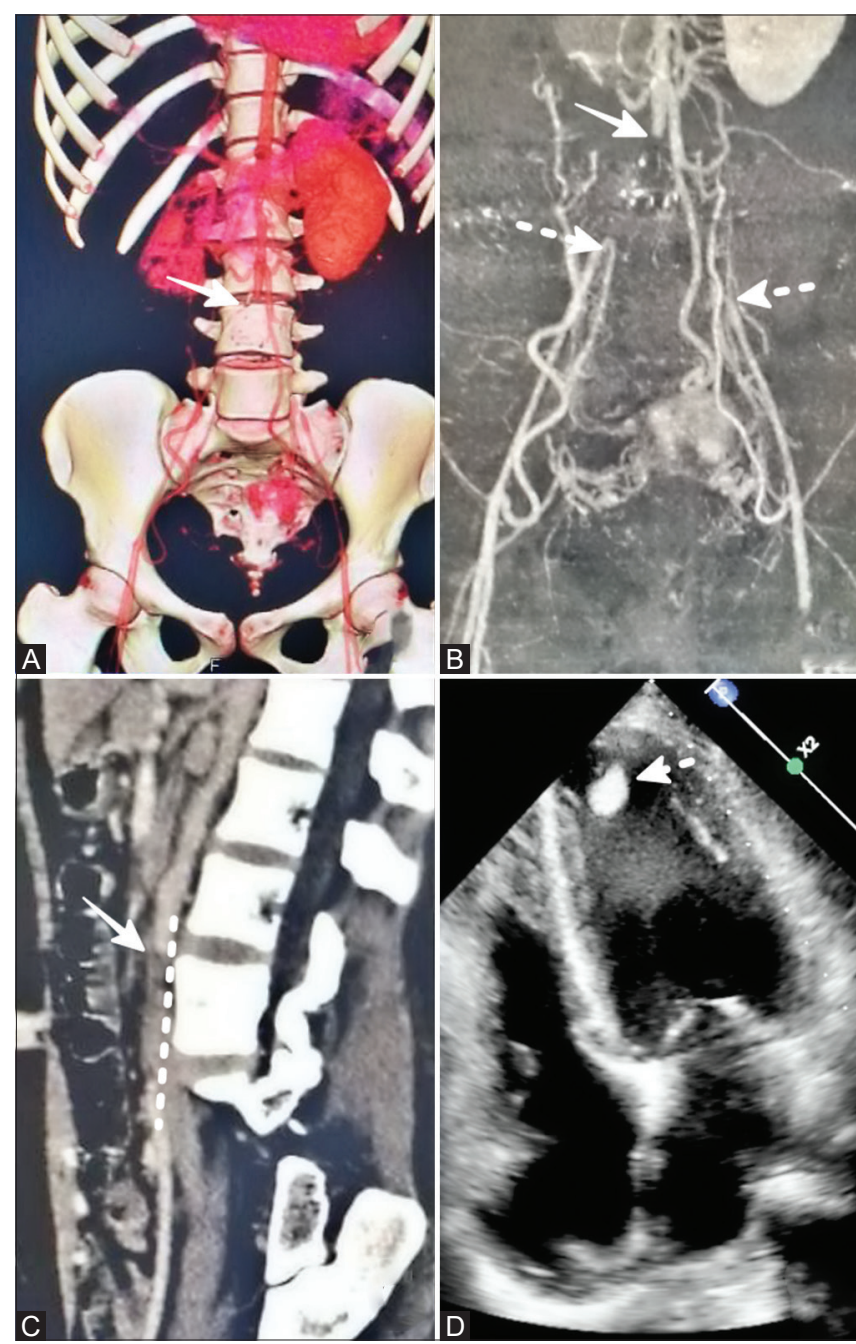

Figure 1 (A-D): (A-D) Computed tomography angiography of the aorta using multiplanar reformatted MPR (A) coronal, (B) sagittal, and (C) SSD images showing complete occlusion of the distal abdominal aorta [solid arrow], bilateral common iliac arteries [dashed arrows]. Bilateral common femoral arteries are filled up by multiple collateral arteries. Collateral vessels are seen arising from the infrarenal abdominal aorta and left renal artery. 2D echocardiography (D) in four-chamber view demonstrated dilated and hypertrophied left ventricle with apical clot [dashed arrow] suggestive of hypertensive heart disease

tapered, while the right iliac artery had a flush occlusion after the origin of the internal iliac artery [Figure 2A and B].

Both iliac arteries initially crossed with Gaia 2 guidewire that entered into the false lumen [Figure 2C and D], then PROGREAT microcatheter and hydrophilic guidewire $(0.018 ")$ system used to re-enter into the true lumen. True lumen entry was confirmed by injection in the abdominal aorta and the visualization of the inferior mesenteric artery branch [Figure 2E and F]. Predilatation of stenotic segments was done using a $4 \times 40 \mathrm{~mm}$ Mustang 0.035 " balloon dilatation catheter system [Boston Scientific Corporation, MA, USA] at 6-8 atm. Angiography of abdominal aorta revealed long-segment stenosis of the infrarenal aorta with osteal occlusion of the right renal artery 


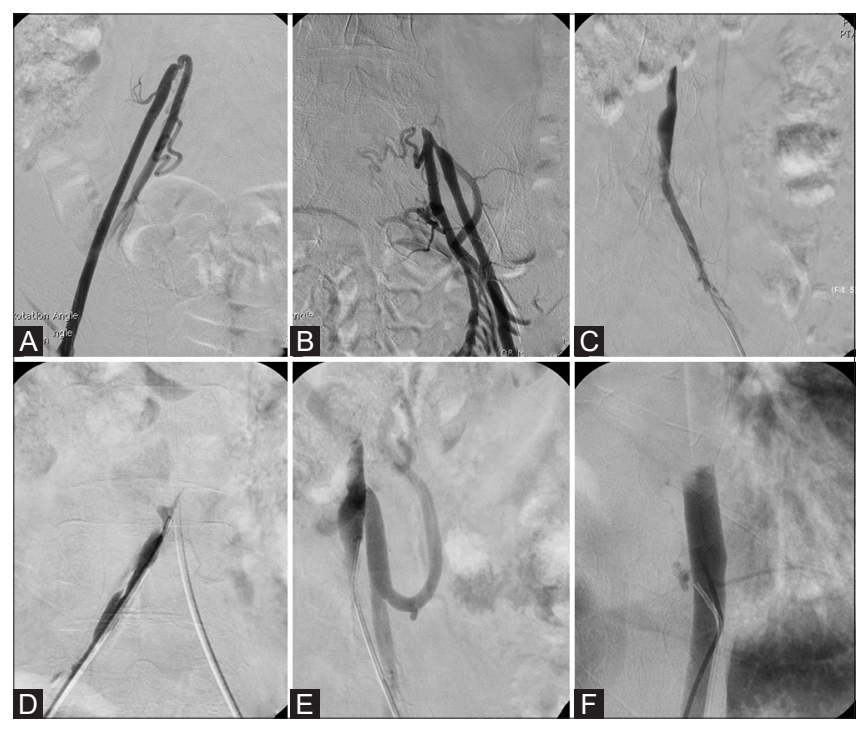

Figure 2 (A-F): (A-F) Digital subtraction angiography showing occlusion of the bilateral common iliac arteries (A and B) after simultaneous retrograde contrast injections done through femoral sheaths. The left and the right common iliac artery was crossed using polymer jacket hydrophilic guidewire that lead to dissection ( $C$ and $D)$. Using PROGREAT Microcatheter and 0.018 hydrophilic guidewire assembly could enter into true lumen that was confirmed by contrast injection into the abdominal aorta and visualization of the inferior mesenteric artery ( $\mathrm{E}$ and $\mathrm{F}$ )

and left renal artery stenosis [Figure 3A]. Bilateral kissing balloon angioplasty of the distal segment of the abdominal aorta and the proximal iliac arteries was performed using two $7 \times 60 \mathrm{~mm}$ Mustang non-compliant balloons [Boston Scientific Corporation, MA, USA] at 8 atm [Figure 3B]. $14 \times 60 \mathrm{~mm}$ Wallstent [Boston Scientific Corporation, MA, USA] was deployed into the abdominal aorta across the renal and other mesenteric arteries to end just before the aortic bifurcation [Figure 3C]. Bilateral $10 \times 80$ and $10 \times 100$ mm LIFESTAR self-expanding Nitinol vascular stents [Bard Peripheral vascular, AZ, USA] for both iliac arteries were deployed simultaneously [Figure 3D].

Final angiography demonstrated brisk flow into the aorta and bilateral iliac arteries. Both peripheral pulses are palpable [Figure 3E and F]. True intraarterial blood pressure was 200/120 $\mathrm{mm} \mathrm{Hg}$ and anti-hypertensive medication doses were escalated. Dual antiplatelet therapy in the form of aspirin and clopidogrel required for at least 12 months but can be extended up to 36 months to prevent restenosis as per the recommendations. She has completed a 1-year follow-up and now in NYHA class I symptoms. In the first year of follow-up, ultrasonography and Doppler study of iliofemoral vessels were performed every 3 months and recommended every 6 months after 1 year to detect early signs of restenosis.

\section{Discussion}

TA is mainly managed by pharmacological therapy but late-stage TA may warrant surgical or endovascular treatment to treat organ ischemia, renovascular hypertension,

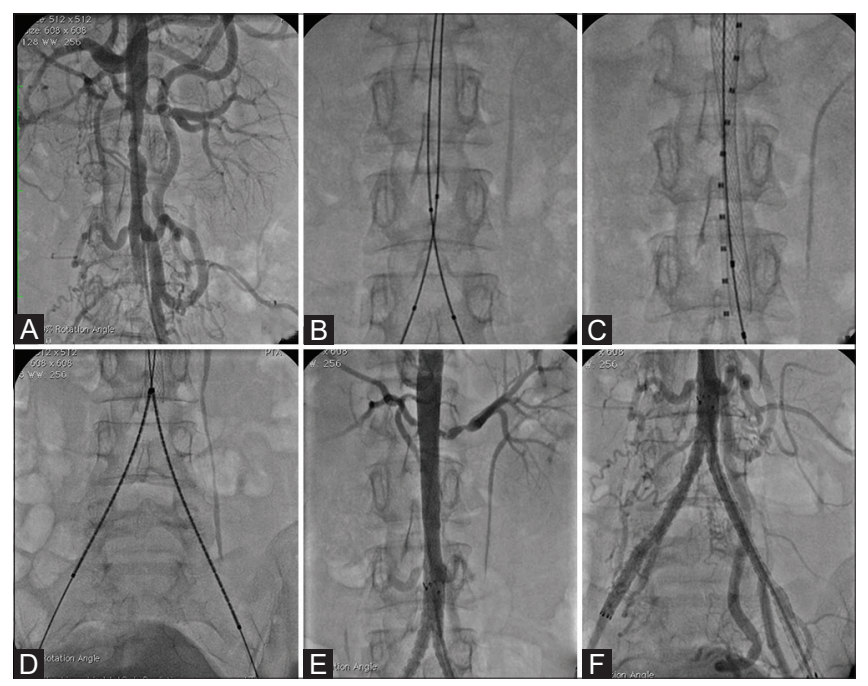

Figure 3 (A-F): (A-F) After connecting from both the common iliac arteries, predilatation of the infrarenal aorta $(A)$, and kissing balloon angioplasty (B) of both CIAs was performed. A calibrated pigtail catheter was placed across the abdominal aorta for the sizing of the stent and Wall stent was deployed (C). Then simultaneous deployment of two self-expanding stents (D) is done. Final angiography demonstrated brisk flow through the well-expanded triple stent with neocarina formation at the aortoiliac bifurcation ( $\mathrm{E}$ and $\mathrm{F}$ )

or aneurysmal lesions. There is, however, a lack of evidence-based consensus about recommendations for surgical or endovascular intervention and appropriate care choices for the patients with TA. ${ }^{[1]}$ However, surgical management was more likely to be accompanied by significant early postoperative complications than endovascular therapy.

In a recent meta-analysis, Jongkind et al. proposed that in patients with the aortoiliac occlusive disease, and endovascular intervention can be successfully executed in specified patients like our case by skilled interveners. Although the primary patency levels tend to be lower than those documented for surgical revascularisation, reinterventions may also be percutaneously performed, resulting in the secondary patency comparable to surgical intervention. ${ }^{[2]}$

Current CT angiography and magnetic resonance angiography (MRA) are less invasive studies and display an equal efficiency in documenting the complete aortoiliac system, the nature of the calcifications, and collateral pathways. As first-line diagnostic methods, they are often favoured when opposed to invasive angiography. Formerly, conventional angiography was the golden standard for demonstrating the complete aortoiliac system, but it is now avoided, due to its invasiveness but performed when there is concurrent scheduled endovascular treatment. ${ }^{[3]}$

Earlier, Fava et al. reported an initial success rate of $100 \%$ in patients with the aortoiliac occlusive disease with TA, with a 5-year patency rate of $60 \%$. Considering the high initial success rate for percutaneous transluminal angioplasty (PTA) in these patients, even though this procedure does not treat 
the disease itself but rather its effects, the disorder recurs in a substantial number of them. ${ }^{[4]}$

A retrospective review of 116 patients with TA is done, who underwent surgery or endovascular repair for over 10 years. Though surgical repairs have shown long-term durability and are suitable for failed endovascular management cases. The study revealed a remarkably similar 1-year patency secondary rate for both the groups of surgical repairs $(98.2 \%)$ and endovascular interventions (98.7\%).

The indications for the endovascular interventions were resistant to renovascular hypertension, refractory hypertension due to aortic stenosis or occlusion, severe claudication of the extremities. The main treatment for endovascular repair was PTA using balloons. Stents were implanted only when repeated angioplasty of the balloon was not clinically effective or when a complication such as dissection or elastic recoil as in our case during PTA. ${ }^{[5]}$

Besides, stenosis caused by arteritis is localized and followed by local fibrous bands, which is distinct from the eccentric stenosis caused by atherosclerosis. Thus it is difficult to reach the specified vessel diameter during most of the dilation therapy. ${ }^{[6]}$ The stenotic portion should be slowly dilated with the non-compliant balloon during stenosis dilation. For the pursuit of better results, an aggressive dilatation should not be executed which could instead of can cause aortic dissection or arterial rupture. The dissection is an intrinsic risk of aortic angioplasty in patients with TA. We eventually made multiple subintimal dissections despite low-pressure predilatation using undersized balloons with the slow rate of inflation. We deployed stents as during the angioplasty as we noticed suboptimal distal runoff. ${ }^{[7]}$

Henry et al. in their experience do not advocate the routine use of the covered stents as they have not provided favourable results in the treatment of athero-occlusive arterial disease.$^{[8]}$ In addition, there are no major studies published about the use of covered stent during angioplasty in TA settings. It is, therefore, generally recommended that their use be limited in the iliofemoral system for aneurysms, arterial ruptures, and arteriovenous fistulas. ${ }^{[9]}$

The appropriate size of the covered stent/stent graft should always accessible on the shelf before a complicated aortoplasty is attempted. We hope that future studies will clarify the correct choice of the balloon (compliant or non-compliant), stents (balloon-expandable or self-expanding, or covered) used for aortoplasty in the TA. ${ }^{[10]}$

Increasingly patients are undergoing endovascular therapy as interventional technologies progress. Nonetheless, large sample-sized randomized controlled trials to determine the benefits and drawbacks of these two treatment strategies are challenging due to its low incidence. Also, there was no consensus or guidelines on the global implementation of treatment approaches suitable for the type of lesion.

\section{Conclusion}

Endovascular therapy is a very well-established technique in clinical settings for aortoiliac artery disease with TA. Angioplasty is also associated with reduced periprocedural morbidity and mortality rates. In comparison, surgery often offers greater long-term patency, while percutaneous therapy can have a late failure but can still be successfully treated with reintervention.

\section{Declaration of patient consent}

The authors certify that they have obtained all appropriate patient consent forms. In the form the patient(s) has/have given his/her/their consent for his/her/their images and other clinical information to be reported in the journal. The patients understand that their names and initials will not be published and due efforts will be made to conceal their identity, but anonymity cannot be guaranteed.

\section{Financial support and sponsorship}

Nil.

\section{Conflicts of interest}

There are no conflicts of interest.

\section{References}

1. Kim YW, Kim DI, Park YJ, Yang SS, Lee GY, Kim DK, et al. Surgical bypass vs endovascular treatment for patients with supra-aortic arterial occlusive disease due to Takayasu arteritis. J Vasc Surg [Internet] 2012;55:693-700.

2. Konstantinos F. Severe aorto-iliac occlusive disease: Options beyond standard aorto-bifemoral bypass. Arch Vasc Med 2018;2:20-4.

3. Mesurolle B, Qanadli SD, El Hajjam M, Goeau-Brissonnière OA, Mignon F, et al. Occlusive arterial disease of the abdominal aorta and lower extremities: Comparison of helical CT angiography with transcatheter angiography. Clin Imaging 2004;28:252-260.

4. Fava MP, Foradori GB, García CB, Cruz FO, Aguilar JG, Kramer AS, et al. Percutaneous transluminal angioplasty in patients with Takayasu arteritis: Five-year experience. J Vasc Interv Radiol [Internet] 1993;4:649-52.

5. Diao Y, Yan S, Premaratne S, Chen Y, Tian X, Chen Z, et al. Surgery and endovascular management in patients with Takayasu's arteritis: A Ten-Year Retrospective Study. Ann Vasc Surg 2020;63:34-44.

6. Lee GY, Jeon P, Do YS, Sung K, Kim DI, Kim YW, et al. Comparison of outcomes between the endovascular treatment and bypass surgery in Takayasu arteritis. Scand J Rheumatol 2014;43:153e61.

7. Damera SR, Barik R. Aorta in Takayasu's arteritis is as brittle as glass: A case report. Vasc Dis Manag 2017;14:E123-6.

8. Henry M, Klonaris C, Amor M, Henry I, Tzvetanov K, Krajcer Z. Which stent for which lesion in peripheral interventions? Tex Heart Inst J 2000;27:119-26.

9. Perera AH, Mason JC, Wolfe JH. Takayasu arteritis: Criteria for surgical intervention should not be ignored. Int J Vasc Med 2013; 2013:618910.

10. Singh N, Athwani V, Bansal V, Kundra S. Takayasu's aorto-arteritis: Not your regular lesion for angioplasty. Ann Pediatr Cardiol 2018;11:312-4. 